\title{
THE TRIANGLE CONDITION FOR PERCOLATION
}

\author{
TAKASHI HARA AND GORDON SLADE
}

\begin{abstract}
Aizenman and Newman introduced an unverified condition, the triangle condition, which has been shown to imply that a number of percolation critical exponents take their mean field values, and which is expected to hold above six dimensions for nearest neighbour percolation. We prove that the triangle condition is satisfied in sufficiently high dimensions for the nearest neighbour model, and above six dimensions for a class of "spread-out" models. The proof uses an expansion which is related to the lace expansion for self-avoiding walk.
\end{abstract}

Percolation is a simple probabilistic model for which many interesting problems remain unsolved. The basic objects in percolation are random graphs on an infinite lattice. Typically there is a critical density for the graph edges (known as bonds) below which there is zero probability that a fixed point in the lattice is part of an infinite connected graph, but above which this probability is strictly positive. This abrupt change in behaviour has been used in chemistry and statistical physics to model phase transitions in a variety of physical systems, such as fluid flow through a porous medium (hence the name percolation), random resistor networks, and the gelation of branched polymers. For an introduction, see [15, 20].

To define the models we are interested in, we consider the $d$-dimensional integer lattice $\mathbf{Z}^{d}$ (whose elements are referred to as sites) and the set of pairs $b=\{x, y\}$ of distinct sites (the bonds). To each bond $b$ is associated an independent Bernoulli random variable $n_{b}$, with $n_{b}=1$ with probability $p \cdot J_{b}$ and $n_{b}=0$ with probability $1-p \cdot J_{b}$. The $J_{b}$ are fixed and $\mathbf{Z}^{d}$ invariant, and $p$ is a parameter. The nearest-neighbour model is defined by taking $J_{b}=1$ for those $b=\{x, y\}$ such that $\|x-y\|_{2}=1$, and $J_{b}=0$ otherwise. Expectation with respect to the joint distribution of the $\left\{n_{b}\right\}$ is denoted by $\langle\cdots\rangle_{p}$.

If $n_{b}=1$ we say $b$ is occupied, and otherwise $b$ is vacant. Given a bond configuration $\left\{n_{b}\right\}$, two sites $x$ and $y$ are said to be connected if there is a path from $x$ to $y$ which consists of occupied bonds. The set of sites which are connected to $x$ is called the connected cluster of $x$ and is denoted $C(x)$. The probability that $x$ is connected to $y$ is written $\tau_{p}(x, y)$,

$$
\tau_{p}(x, y)=\langle I[x \text { and } y \text { are connected }]\rangle_{p}=\langle I[y \in C(x)]\rangle_{p},
$$

where $I$ denotes the indicator function. Denoting by $|C(x)|$ the number of sites in $C(x)$, the expected cluster size or susceptibility is given by

$$
\chi(p)=\sum_{x} \tau_{p}(0, x)=\langle|C(0)|\rangle_{p} .
$$

Received by the editors January 31, 1989 and, in revised form, March 15, 1989.

1980 Mathematics Subject Classification (1985 Revision). Primary 82A43, 60K35.

(c) 1989 American Mathematical Society $0273-0979 / 89 \$ 1.00+\$ .25$ per page 
The percolation density $P_{\infty}$ is defined to be the probability that the origin is connected to an infinite cluster

$$
P_{\infty}(p)=\langle I[|C(0)|=\infty]\rangle_{p} .
$$

If $P_{\infty}>0$, then percolation is said to occur.

It has been known for thirty years that under quite general conditions, for general dimension $d \geq 2$, there is a critical value $p_{c} \in(0,1)$ such that $P_{\infty}(p)=0$ for $p<p_{c}$ and $P_{\infty}(p)>0$ for $p>p_{c}[8,11]$. Such a critical point exists in particular if $0<\sum_{x} J_{\{0, x\}}<\infty$. It has recently been shown that $p_{c}$ can also be characterized by $p_{c}=\sup \{p: \chi(p)<\infty\}[16,2]$. By analogy with other statistical mechanical models, and in agreement with numerical simulations, $\chi$ and $P_{\infty}$ are expected to satisfy power laws near $p_{c}$ of the form

$$
\begin{array}{cl}
\chi(p) \sim\left(p_{c}-p\right)^{-\gamma} & \text { as } p \nearrow p_{c} \\
P_{\infty}(p) \sim\left(p-p_{c}\right)^{\beta} & \text { as } p \searrow p_{c} .
\end{array}
$$

The precise interpretation of (2) is that there are positive constants $c_{1}$ and $c_{2}$ such that $c_{1}\left(p_{c}-p\right)^{-\gamma} \leq \chi(p) \leq c_{2}\left(p_{c}-p\right)^{-\gamma}$ for $p<p_{c}$, and similarly for (3). In addition to $\gamma$ and $\beta$ other critical exponents can be defined, such as $\delta$, defined by

$$
M\left(p_{c}, h\right) \sim h^{1 / \delta} \text { as } h \searrow 0
$$

where $M(p, h)=1-\sum_{1 \leq n<\infty} e^{-h n}\langle I[|C(0)|=n]\rangle_{p}$ and the $\sim$ is interpreted as in (2). Formally $\delta$ is related to the decay rate of $\langle I[|C(0)|=n]\rangle_{p_{c}}$ as $n \rightarrow \infty$.

The existence of these critical exponents remains unproved, apart from the results stated below. The critical exponents are expected to be dimension dependent in low dimensions, but are expected not to depend on the short-range behaviour of the $J_{b}$ or on the structure of the lattice. This lack of dependence on the $J_{b}$ or the lattice is known as universality, and different models which lead to the same critical exponents are said to be in the same universality class. In addition, it is believed that for the nearest neighbour percolation model for $d>6$, all critical exponents are also dimension independent and take their so-called mean-field values, i.e., the corresponding values for percolation on the Bethe lattice (the infinite tree with all vertices having the same number (at least three) of branches), also known as the Cayley tree. On the Bethe lattice it is not difficult to prove the above power law behaviour, with $\gamma=1, \beta=1, \delta=2$. On the other hand, it is known that for $d<6$ it is not possible for all critical exponents (assuming they exist) to simultaneously take their mean-field values $[10,21]$, and hence the upper critical dimension, i.e., the dimension above which mean-field behaviour takes over, is expected to be six.

Aizenman and Newman [4] introduced an unverified condition, the triangle condition, which is expected to hold above six dimensions for models in the universality class of the nearest neighbour model, and which was shown by them to imply that (2) holds with $\gamma=1$. This condition is analogous to the finiteness of the "bubble diagram" for Ising, $\varphi^{4}[1]$, 
and self-avoiding walk [7] models. Subsequently Barsky and Aizenman [5] showed that the triangle condition implies that (3) and (4) hold with $\beta=1$ and $\delta=2$. Related results were obtained by Nguyen [17] for the so-called gap exponents. In particular, $(3)$ implies that $P_{\infty}\left(p_{c}\right)=0$, which has been proved before now only for the two dimensional nearest neighbour model [18].

We prove that the triangle condition is satisfied in two situations, and hence the above consequences of the triangle condition follow. To state the triangle condition we first define the "triangle diagram" $\nabla(p)$, which is defined in terms of the two-point function $\tau_{p}(x, y)$ of equation (1),

$$
\nabla(p)=\sum_{x, y} \tau_{p}(0, x) \tau_{p}(x, y) \tau_{p}(y, 0) .
$$

It is useful to write the triangle diagram also in terms of the Fourier transform

$$
\hat{\tau}_{p}(k)=\sum_{x} \tau_{p}(0, x) e^{i k \cdot x} .
$$

Using the fact that the Fourier transform of a convolution is a product of Fourier transforms,

$$
\nabla(p)=\int_{[-\pi, \pi]^{d}} \frac{d^{d} k}{(2 \pi)^{d}} \hat{\tau}_{p}(k)^{3} .
$$

The triangle condition states that $\nabla\left(p_{c}\right)<\infty$. This statement is not without content, as it is known that $\hat{\tau}_{p}(0)=\chi(p) \rightarrow \infty$ as $p \nearrow p_{c}$ [4].

THEOREM 1. The triangle condition and the infrared bound $\hat{\tau}_{p}(k) \leq$ const. $k^{-2}$, uniformly in $p<p_{c}$, hold in the following situations: (a) for $d \geq 92$ for the nearest neighbour model, and (b) for $d>6$ and $J_{\{0, x\}}=$ $L^{-d} g(x / L)$, if $L$ is sufficiently large and $g: \mathbf{R}^{d} \rightarrow[0, \infty)$ is $\mathbf{Z}^{d}$-invariant, $e^{\delta\|x\|_{2}} g \in L^{\infty}$ for some $\delta>0$, and all first order partial derivatives of $g$ with respect to the $x_{\mu}$ (considered as distributions) are integrable.

A basic example for (b) is $g(x)=1$ if $\|x\|_{\infty} \leq 1$, and $=0$ otherwise. The dimension 92 in $(a)$ can doubtless be reduced, but a new idea will be needed to lower it all the way down to six. However, since the models in (a) and (b) are expected to be in the same universality class, (b) supports the conjecture that the triangle condition is also satisfied for the nearest neighbour model in more than six dimensions. Similar methods can be used to prove that the critical exponent $\nu$ for the correlation length equals $1 / 2$ in (a) and (b) [12], and to study lattice trees and lattice animals above the expected upper critical dimension of eight [14]. We expect these methods can also be used to prove that the triangle condition is satisfied above three dimensions for a "spread-out" version of percolation with $J_{\{x, y\}} \sim\|x-y\|_{2}^{-2}$.

The details of the proof will appear in [13]. Here we give a very brief outline of some of the important ideas in the proof of (a). The proof of 
(b) is similar. We define

$$
\begin{gathered}
T(p)=\nabla(p)-1=\sum_{x, y} \tau_{p}(0, x) \tau_{p}(x, y) \tau_{p}(y, 0)-1 \\
W(p)=\sum_{x}|x|^{2} \tau_{p}(0, x)^{2} .
\end{gathered}
$$

We also introduce the gaussian quantities $T_{G}$ and $W_{G}$ defined by replacing $\tau_{p}$ by the massless gaussian propagator

$$
C(x, y)=\int_{[-\pi, \pi]^{d}} \frac{d^{d} k}{(2 \pi)^{d}} e^{i k \cdot(x-y)}\left[1-\frac{1}{d} \sum_{\mu=1}^{d} \cos k_{\mu}\right]^{-1}
$$

in (5) and (6). It can be shown that there are constants $K_{T}$ and $K_{W}$ such that $T_{G} \leq K_{T} / d$ and $W_{G} \leq K_{W} / d$, for $d \geq 6+\varepsilon$. The proof of (a) is accomplished by showing that the following three statements hold.

(i) For $p<p_{c}, T(p)$ and $W(p)$ are continuous functions of $p$.

(ii) For $p \leq(2 d)^{-1}, T(p) \leq T_{G}$ and $W(p) \leq W_{G}$.

(iii) Let $d$ be sufficiently large and fix any $p \in\left[1 / 2 d, p_{c}\right)$. If $T(p) \leq$ $4 K_{T} d^{-1}$ and $W(p) \leq 4 K_{W} d^{-1}$ then in fact $T(p) \leq 2 K_{T} d^{-1}$ and $W(p) \leq$ $2 K_{W} d^{-1}$.

It then follows from(i)-(iii) that for $p<p_{c}$ the graphs of $T(p)$ and $W(p)$ must lie below $2 K_{T} d^{-1}$ and $2 K_{W} d^{-1}$. It is not difficult to show (using the monotonicity and continuity of $\tau_{p}$ in $p$ [3]) that this uniform bound on $T(p)$ for $p<p_{c}$ implies the same bound for $T\left(p_{c}\right)$, and hence by definition of $T$ the triangle condition holds if $d$ is sufficiently large.

The continuity in (i) follows from the continuity of the two-point function in $p$ [3] and the monotone convergence theorem, and the proof of (ii) is elementary. The proof of (iii) (whose statement has been simplified here-the complete statement will appear in [13]) is the significant part of the analysis. For the self-avoiding walk the analogue of step (iii) was obtained in [19] using the lace expansion [9]. For percolation we derive and use a related expansion, which is based on the inclusion-exclusion relation. The derivation of the expansion hinges on the concept of the pivotal bond, and convergence of the expansion is obtained by taking $d^{-1}$ small. The van den Berg-Kesten inequality [6] plays the role played by the repulsive interaction for the self-avoiding walk.

Acknowledgements. We thank Michael Aizenman, David Brydges, Chuck Newman, Alan Sokal and Hal Tasaki for valuable comments and stimulating discussions, and Fred Hoppe and Neal Madras for their comments on an earlier version of this announcement. This work was supported in part by the Nishina Memorial Foundation (T.H.), NSF grant PHY-8896163 (T.H.), and NSERC grant A9351 (G.S.).

\section{REFERENCES}

1. M. Aizenman, Geometric analysis of $\varphi^{4}$ fields and Ising models, Comm. Math. Phys. 86 (1982), 1-48.

2. M. Aizenman and D. J. Barsky, Sharpness of the phase transition in percolation models, Comm. Math. Phys. 108 (1987), 489-526. 
3. M. Aizenman, H. Kesten and C. M. Newman, Uniqueness of the infinite cluster and continuity of connectivity functions for short and long range percolation, Comm. Math. Phys. 111 (1987), 505-531.

4. M. Aizenman and C. M. Newman, Tree graph inequalities and critical behaviour in percolation models, J. Statist. Phys. 36 (1984), 107-143.

5. D. Barsky and M. Aizenman, Percolation critical exponents under the triangle condition, preprint (1988).

6. J. van den Berg and H. Kesten, Inequalities with applications to percolation and reliability, J. Appl. Prob. 22 (1985), 556-569.

7. A. Bovier, G. Felder and J. Fröhlich, On the critical properties of the Edwards and the self-avoiding walk model of polymer chains, Nucl. Phys. B230 [FS210] (1984), 119-147.

8. S. R. Broadbent and J. M. Hammersley, Percolation processes. I. Crystals and mazes, Proc. Cambridge Philos. Soc. 53 (1957), 629-641; J. M. Hammersley: Percolation processes. II. The Connectivity constant, ibid, 642-645.

9. D. Brydges and T. Spencer, Self-avoiding walk in 5 or more dimensions, Comm. Math. Phys. 97 (1985), 125-148.

10. J. T. Chayes and L. Chayes, On the upper critical dimension of Bernoulli percolation, Comm. Math. Phys. 113 (1987), 27-48.

11. J. M. Hammersley, Bornes supérieures de la probabilité critique dans un processus de filtration, Le Calcul des Probabilités et ses Applications, CNRS, Paris, 1959, pp. 17-37.

12. T. Hara, Mean field critical behaviour of correlation length for percolation in high dimensions (in preparation).

13. T. Hara and G. Slade, Mean field critical phenomena for percolation in high dimensions, preprint (1989). tion).

14.

15. H. Kesten, Percolation theory and first passage percolation, Ann. Probab. 15 (1987), 1231-1271.

16. M. V. Menshikov, S. A. Molchanov and A. F. Sidorenko, Percolation theory and some applications, Itogi Nauki i Tekhniki (Series of Probability Theory, Mathematical Statistics, Theoretical Cybernetics) 24 (1986), 53-110; English Translation: J. Soviet Math. 42 (1988), 1766-1810.

17. B. G. Nguyen, Gap exponents for percolation processes with triangle condition, J. Statist. Phys. 49 (1987), 235-243.

18. L. Russo, On the critical percolation probabilities, Z. Wahrsch. Verw. Gebiete 56 (1981), 229-237.

19. G. Slade, The diffusion of self-avoiding random walk in high dimensions, Comm. Math. Phys. 110 (1987), 661-683.

20. D. Stauffer, Introduction to percolation theory, Taylor and Francis, London and Philadelphia, 1985.

21. H. Tasaki, Hyperscaling inequalities for percolation, Comm. Math. Phys. 113 (1987), 49-65.

Courant Institute of Mathematical Sciences, New York University, 251 Mercer STREeT, NeW York, NeW YORK 10012

\footnotetext{
Department of Mathematics and Statistics, McMaster University, Hamilton, Ontario, Canada L8S 4K1
} 
